\title{
IMMUNOLOGICAL STUDIES OF THE SPERM-SPECIFIC PHOSPHOGLYCERATE KINASE-2 OF MICE
}

\author{
ROBERT P. ERICKSON ${ }^{1,2}$, KENNETH HARPER $^{1}{ }^{1}$, ALAN MENGE $^{3}$ and CHI-YU LEE ${ }^{4}$ \\ ${ }^{1}$ Department of Human Genetics, ${ }^{2}$ Pediatrics and ${ }^{3}$ Obstetrics and Gynecology, University of \\ Michigan School of Medicine, Ann Arbor, MI 48109, and ${ }^{4}$ Laboratories of Environmental \\ Mutagenesis and Biochemical Genetics, National Institute of Environmental Health Sciences, \\ Research Triangle Park, NC 27709, U.S.A.
}

(Received 16 May 1979; accepted 15 June 1979)

\begin{abstract}
Antiserum was prepared to 500-fold purified PGK-2A from mouse testes. Despite the apparent high purity of the immunizing antigen, double diffusion analyses showed two precipitin lines, one of which can be shown to correspond to PGK-2. An immunoabsorbent column of liver homogenate coupled to cyanogen bromide-activated Sepharose was used to remove the contaminating antibody. Both immuno-inactivation and double diffusion analyses demonstrated that the PGK-2 antigen appeared in testes between days 30 and 34 of postnatal development. The antisera prepared against highly purified PGK-2A did not disclose any immunological differences between PGK-2A, PGK-2B, and PGK-2C either by double diffusion analyses or quantitative immunoprecipitation. Antisera to PGK-2 did not inhibit in vitro fertilization of zona-free hamster ova by mouse spermatozoa.
\end{abstract}

\section{INTRODUCTION}

Two isoenzymes of 3-phosphoglycerate kinase (ATP:3-phospho-D-glycerate 1-phosphotransferase, EC 2.7.2.3, PGK) are known to be present in mammalian tissues. PGK-1 is found in all somatic tissues and the structural locus is X-linked in humans (Chen et al., 1971), kangaroos (Cooper et al., 1971), and mice (Nielsen and Chapman, 1977). PGK-2 has been detected only in the sperm and testes of a number of mammals; however, there are exceptions where it has been found in somatic cells (kangaroo, dog and fox; Vandenberg et al., 1973). PGK-2 has previously been purified (Pegaro and Lee, 1978) and antisera have been prepared to the highly purified protein. Although PGK-2 is controlled by an autosomal locus, it was found to be biochemically very similar to the X-linked PGK-1 and a slight immunological cross reaction was found (Pegaro et al., 1978). PGK-2 is reported to be one of a few enzymes which appear only after meiosis, i.e., during spermiogenesis. Vandenberg et al. (1973) could not detect a band of PGK-2 by starch gel electrophoresis of testes extracts until mice were 22 days of age and substantial activity was not found until day 30. This appearance of PGK-2 on day 22 corresponds to the first appearance of spermatids in most inbred strains of mice (Nebel et al., 1961) and a postmeiotic time of synthesis seemed likely. However, PGK-2 might be present as a precursor but not be electrophoretically detectable before 22 days. Therefore the appearance of PGK-2 was studied by immunological methods which could detect inactive enzymatic molecules. 
Three variants of PGK-2 (PGK-2A, PGK-2B, PGK-2C) are known in inbred strains of mice. PGK-2C is a low activity variant which is possibly related to decreased fertility. These variants allow the mapping of the structural locus for PGK-2 to a site very near the H-2 locus on chromosome 17 (Eicher et al., 1978). Therefore the immunological relationships of the three PGK-2 variants were also studied. We also sought to determine if the antisera to this sperm-specific enzyme would inhibit in vitro fertilization since this has been shown to occur with antisera to LDH-X (Erickson et al., 1975b).

\section{MATERIALS AND METHODS}

\section{Preparation of antisera}

PGK-2A was purified as previously described (Pegaro and Lee, 1978) and antisera were prepared in rabbits by several protocols (Pegaro et al., 1978). We have used antisera II of that report which inactivates PGK-2 but not PGK-1 .

\section{Enzyme inactivation}

Aliquots of testes homogenates (1:10 w/v 0.005 M EDTA) from mice of various ages were incubated with increasing amounts of heat-inactivated sera in a final volume of $100 \mu \mathrm{l}$ for $30 \mathrm{~min}$ at room temperature. The samples were then centrifuged at $12000 \mathrm{~g}$ for $10 \mathrm{~min}$ and supernatants immediately assayed for residual PGK activity according to the procedure described in Pegaro and Lee (1978). Incubations with the same amount of normal rabbit sera were always performed in parallel and the activity remaining corrected for any loss of activity due to non-specific factors involved in the incubation procedure.

\section{Immunoprecipitation studies}

Double immunodiffusion tests were performed using Hyland Laboratories prepared plates. Quantitative immunoprecipitation was performed by incubating $100 \mu \mathrm{g}$ of the purified enzyme with increasing volumes of antisera in a final total volume of $0.5 \mathrm{ml}$. After $30 \mathrm{~min}$ at room temperature and overnight at $4^{\circ} \mathrm{C}$, the reaction mixture was centrifuged for $30 \mathrm{~min}$ at $1400 \mathrm{~g}$. The supernatant was discarded and pellets were washed twice with phosphate-buffered saline with centrifugation as above. The final pellet was dissolved in $0.1 \mathrm{ml}$ of normal sodium hydroxide and the $\mathrm{OD}_{280}$ was determined.

\section{Preparation of immobilized liver homogenate on Sepharose}

Ten grams of frozen liver were homogenized in $20 \mathrm{ml}$ of $0.2 \mathrm{M} \mathrm{NaHCO}_{3}$ at $\mathrm{pH} 7.8$. After centrifugation for 20 min at $27000 \mathrm{~g}, 5 \mathrm{ml}$ of supernatant was mixed with $10 \mathrm{~g}$ of $\mathrm{CNBr}$-activated Sepharose and shaken vigorously at $4^{\circ} \mathrm{C}$ for $5 \mathrm{~h}$. After coupling, the liverSepharose was washed thoroughly with $100 \mathrm{ml}$ of $0.2 \mathrm{M} \mathrm{NaHCO}_{3}$.

\section{Purification of anti-PGK-2 with immobilized liver homogenate}

Twenty $\mathrm{ml}$ of antiserum to PGK-2 were passed through a column which contained $10 \mathrm{ml}$ of liver-Sepharose which had been equilibrated with $0.2 \mathrm{M} \mathrm{NaHCO}_{3}, \mathrm{pH} 7.8$. The 
unadsorbed antisera were eluted with the same buffer and pressure-concentrated until the protein concentration was the same as that of the original serum.

\section{In vitro fertilization of zona-free hamster eggs}

Sperm expressed from the caudal epididymides of a mouse were incubated for $2 \mathrm{~h}$ at $37^{\circ} \mathrm{C}$ under $5 \% \mathrm{CO}_{2}$ in air in $2 \mathrm{ml}$ of a modified Krebs--Ringer bicarbonate solution (Yoyoda and Chang, 1974). After the incubation period, the sperm concentration was adjusted to approximately $2 \times 10^{5}$ per $\mathrm{ml}$ with fresh medium and $0.4 \mathrm{ml}$ aliquots were prepared in $38 \mathrm{~mm}$ watchglasses. After the addition of $10 \%$ control and immune rabbit sera, the sperm samples were covered with mineral oil and incubated for $30 \mathrm{~min}$. Zonafree hamster ova, prepared by the method of Yanagamachi et al. (1976), were then added to the sperm-serum samples. After an additional $4 \mathrm{~h}$ incubation, the ova were washed twice in fresh medium to remove unbound and loosely bound sperm and then examined under a phase-interference microscope (Nachet). Evidence of sperm penetration was the occurrence of one or more swollen sperm heads within the ova.

\section{RESULTS}

\section{Specificity of antisera and time of appearance of antigenic PGK-2 in testes}

The immuno-inactivation of phosphoglycerate kinase activity by the anti-PGK-2 antisera was performed on extracts of mice of several ages (Fig. 1) near the first wave of meiosis. Even at the highest concentration of antiserum used, the activity of PGK for ages 25 days and younger was not reduced, while at the highest concentration of antiserum, PGK-2 activity was reduced $47 \%$ at 34 days and $85 \%$ at 36 days. For both 36 and 34 days increasing concentrations of antiserum resulted in increasing loss of activity. This result demonstrates the rapid accumulation of PGK-2 in developing testes between postnatal ages of 31 and 36 days.

We also studied the time of appearance of PGK-2 by double diffusion. As seen in Fig. 2 there is a component of the antisera which reacts with testes extracts from mice of all ages. However, a second line was clearly seen which created a spur over the first line in day 34 testes but not in day 28 or day 23 testes. Thus, there is a component of the sera which will precipitate PGK-2 and this activity does not appear in testes until after day 28 during postnatal mouse development. The other component of the antisera reacted with liver and kidney as well as with immature testes and could be removed by immunoabsorption of the antisera with immobilized liver homogenate (Fig. 3).

\section{Immunological relatedness of $P G K-2 A, P G K-2 B$, and $P G K-2 C$}

A preparation of highly purified PGK-2A (similar to that to which the antisera were raised) and 30- to 40-fold, partially purified, PGK-2B and PGK-2C were studied in double diffusion and compared to adult and 13 day testes. The unabsorbed serum was used as there was a slight decrease in degree of reactivity with absorption. As seen in Fig. 4, only the very highly purified PGK-2A showed none of the precipitation arc which fuses with 


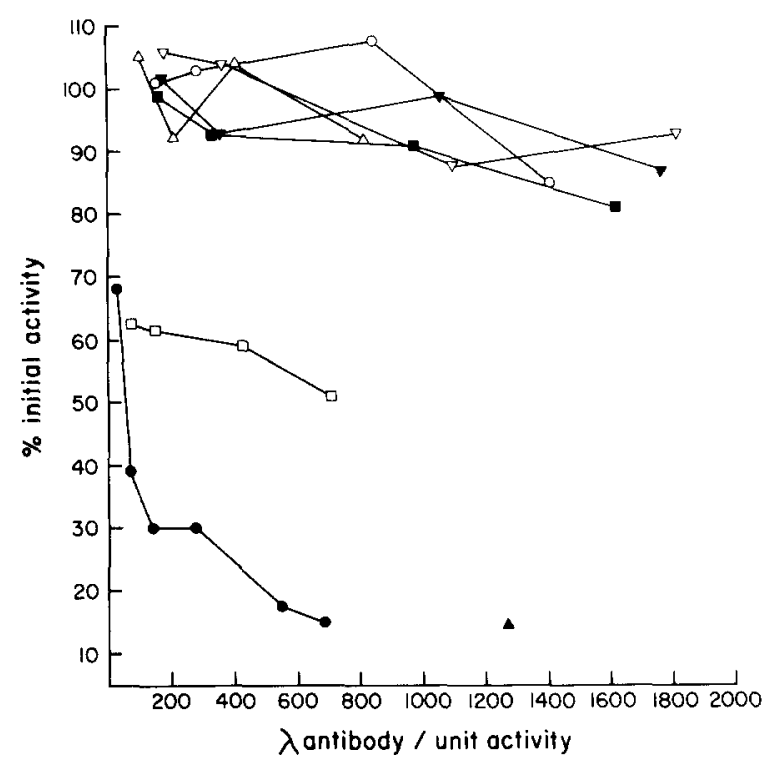

Fig. 1. Immuno-inactivation of total PGK from testes of mice of different ages. Increasing amounts of antibody were incubated with testes extracts and the residual activity measured as described in Materials and Methods. The percent inactivation is plotted on the ordinate and the $\mu$ l of antibody per unit of initial activity on the abscissa: $\bullet$, adult testes; $\bullet$, 36-day; •, 34-day; $\nabla, 31$-day; $\nabla, 25$-day; $\bullet$, 22-day; $\triangle, 19$-day; $\circ$, 13-day.

extracts of 13-day testes, the latter indicating a contaminating antigen. This arc which is seen in 13-day testes is also seen in adult testes, PGK-2B and PGK-2C. A second arc, which is the only arc seen with the preparation of PGK-2A, fuses with PGK-2C, adult testes and PGK-2B. There are no visible spurs of any of these PGKs over each other. Thus, PGK-2A, 2B and 2C seem identical when this serum is used in double diffusion.

Quantitative immunoprecipitation of PGK-2C and PGK-2A was also performed. The PGK-2C preparation, which was used in the double diffusion above and which did contain a contaminating antigen, showed a maximum precipitation at $1 \mu$ l of antibody per microgram of antigen (Fig. 5). At $2 \mu \mathrm{l}$ of antibody per microgram of antigen the amount of precipitate had been decreased by about 50\%. PGK-2A was still increasing in amount of immunoprecipitate at a ratio of $4 \mu$ of antibody to microgram of antigen. The rate of increase in quantity of immunoprecipitate with increasing amounts of antibody paralleled that seen with PGK-2C but was shifted to higher ratios of antibody to antigen. This is in accord with the greater purity of the PGK-2A and suggests that maximal precipitation or its sequel, an inhibition of precipitation, had not been achieved at ratios of antibody to antigen which were practicable. Thus, there was no evidence of antigenic differences between PGK-2A and PGK-2C by a quantitative immunoprecipitation.

\section{The effects of antisera to $P G K-2$ on fertility}

We studied the effects of several of the antisera previously described (Pegora et al., 1978) on in vitro fertilization of zona-free hamster eggs by mouse spermatozoa (Table 1). 

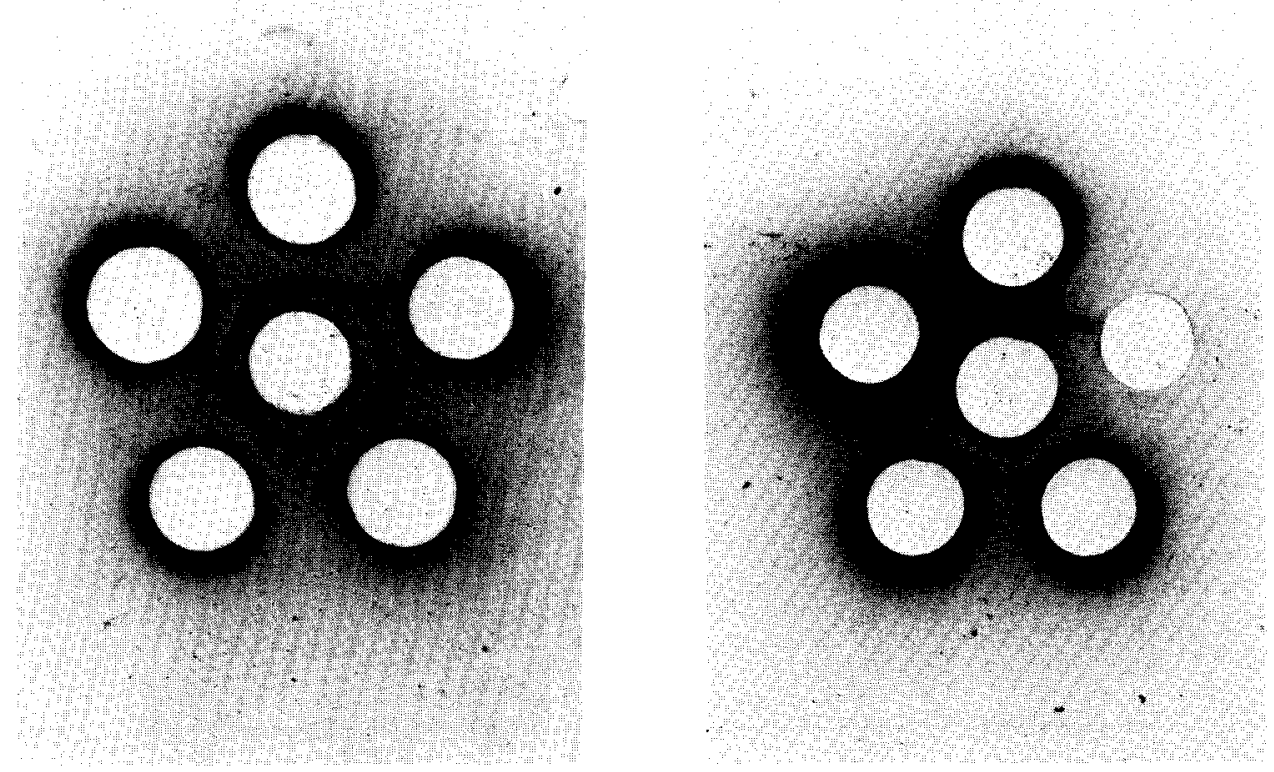

Fig. 2. Double diffusion analysis of testes homogenates of mice of various ages with antiserum to PGK-2. Center well: antiserum to PGK-2; 12 o'clock, adult testes; 2 o'clock, 23-day testes; 5 o'clock, 34-day testes; 7 o'clock, adult testes; 10 o'clock, 28-day testes.

Fig. 3. Double diffusion analysis of liver, kidney and testes homogenates with absorbed antiserum to PGK-2; 12 o'clock, adult testes; 2 o'clock, adult kidney; 5 o'clock, 22-day testes; 7 o'clock, 13-day testes; 10 o'clock, adult liver.
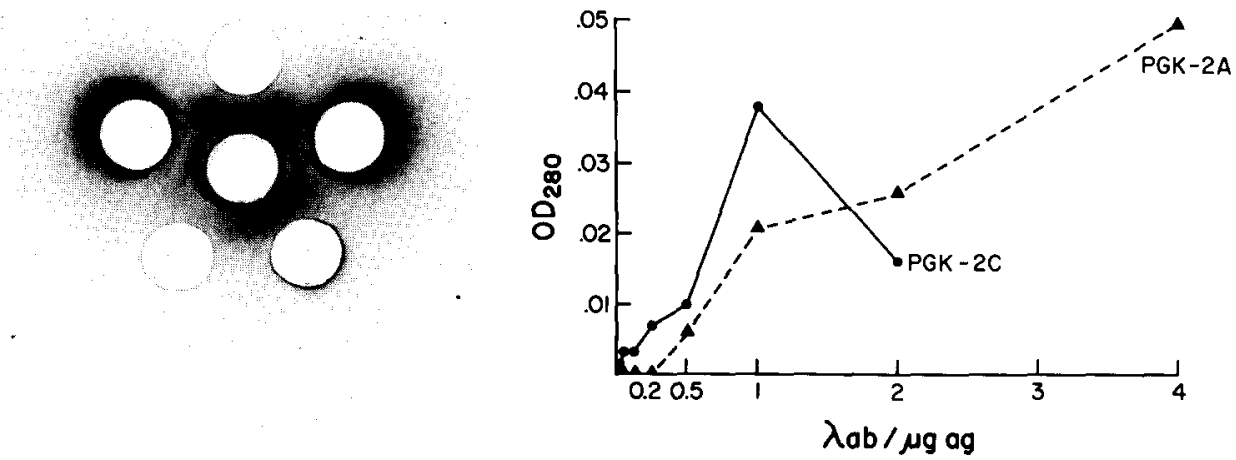

Fig. 4. Double diffusion analysis of purified, partially purified and non-purified preparations of PGK with antiserum to PGK-2 (A). Center well: antiserum to PGK-2A; 12 o'clock, partially purified PGK2B; 2 o'clock, 13-day testes homogenate; 5 o'clock, partially purified PGK-2C; 7 o'clock, highly purified PGK-2A; 10 o'clock, adult testes (PGK-2A).

Fig. 5. Quantitative immunoprecipitation analyses of highly purified PGK-2A and partially purified PGK-2C with antisera to PGK-2A. Increasing quantities of antibody were incubated with the indicated amounts of antigen as described in Materials and Methods. Ordinate: $\mathrm{OD}_{280}$ of dissolved immunoprecipitate. Abscissa: ratio of antibodies to micrograms of antigen. 


\section{TABLE 1}

The effects of anti-Pgk-2 antisera on the ability of mouse spermatozoa to penetrate zona-free hamster ova

\begin{tabular}{|c|c|c|c|c|c|}
\hline \multirow[t]{2}{*}{ Sample } & \multirow[t]{2}{*}{ No. ova } & \multicolumn{2}{|c|}{$\%$ Ova with sperm } & \multicolumn{2}{|c|}{$\%$ Sperm agglutinated } \\
\hline & & Attached & Penetrated * & Agglutinated & Size \\
\hline Media & 4 & 100 & 100 & $<5$ & $2-3$ sperm \\
\hline Anti-Pgk-2I & 10 & 100 & 80 & 60 & Large $(<20)$ \\
\hline Control for I & 10 & 100 & 80 & 20 & Small ( $3-5$ usual) \\
\hline Anti-Pgk-2II & 20 & 100 & 80 & 70 & Large \\
\hline Control for II & 17 & 100 & 76.5 & 20 & Small \\
\hline Anti-Pgk-2III & 10 & 100 & 70 & High & - \\
\hline Control for III & 10 & 100 & 80 & Low & - \\
\hline $\begin{array}{l}\text { Anti-mouse } \\
\text { epididymal } \\
\text { sperm }\end{array}$ & 10 & 80 & 0 & 90 & Large \\
\hline
\end{tabular}

* Swollen heads, pronuclei and tails.

In each case a normal rabbit antiserum was used for a control. Three different antisera with varying titers of PGK-2 inactivating activity were used as $10 \%$ solutions of the sera in the in vitro fertilization media and compared to $10 \%$ control sera. No effect was found. A positive control of anti-mouse epididymal sperm serum was also included. It completely prevented sperm penetration of the zona-free hamster egg. Thus, antisera to PGK-2 have no apparent effect on in vitro fertilization.

\section{DISCUSSION}

In the mouse, presumptive gametes do not undergo meiosis to become spermatids until about day 22 (Nebel et al., 1961). PGK-2 could not be identified with antisera to PGK-2 at day 23 and 28 by double diffusion nor at day 25 and 31 by immuno-inactivation, but could be identified at day 34 by both techniques. Therefore, these new immunological data complement the electrophoretic evidence (Vandenberg et al., 1976) that PGK-2 is expressed postmeiotically in mouse sperm. Although the structural locus for PGK-2 is closely linked to the major histocompatability locus, the time of appearance of PGK-2 is discordant with the time of appearance of $H-2$ region antigens: $H-2 \mathrm{D}$ and $\mathrm{K}$ antigens and Ia antigens appear before meiosis (Erickson, 1977). The three PGK-2 variants, PGK-2A, PGK-2B and PGK-2C, were not distinguishable from each other by double diffusion and PGK-2A and PGK-2C were not distinguishable by quantitative immunoprecipitation. Antigenic differences might have been found since all three differ in electrical charge and heat stability and since it is possible that PGK-2C has a decreased $V_{\text {max }}$ to explain its low level of activity (Pegaro and Lee, 1979).

In general, it would not be expected that antisera to sperm enzymes, thought to be contained within the spermatozoa, should inhibit fertilization. However, Goldberg and collaborators discovered that antisera to LDH.X decreased fertility in mice and rabbits 
up to $50 \%$ (Goldberg and Lerum, 1972). This inhibition seems to be due to the release of LDH-X from some damaged spermatozoa with its secondary absorption onto other intact spermatozoa (Erickson et al., 1975a). This is compatible with the apparent effect of antisera to LDH-X on in vitro fertilization, but not on embryonic survival (Erickson et al., 1975b). Thus, it seems to be an unusual case for an antiserum to an intracellular enzyme to inhibit fertilization and it is the high levels of LDH-X in spermatozoa that probably are responsible for the unexpected findings with antisera to LDH-X and fertility. The lack of effect of antisera to PGK-2 on in vitro fertilization may be a more usual finding with antisera to intracellular spermatozoal enzymes.

\section{REFERENCES}

Chen, S.-H., Malcolm, L.A., Yoshida, A. and Giblett, E.R. (1971) Phosphoglycerate kinase: an $\mathrm{X}$-linked polymorphism in man. Am. J. Hum. Genet. 23, 87-91.

Cooper, D.W., Vandenberg, J.L., Sharman, G.B. and Poole, W.E. (1971) Phosphoglycerate kinase polymorphism in kangaroos provides further evidence for paternal X-inactivation. Nature New Biol. $230,155-157$.

Eicher, E.M., Cherry, M., and Flaherty, L. (1978) Autosomal phosphoglycerate kinase linked to mouse major histocompatibility complex. Mol. Gen. Genet. 158, 225.

Erickson, R.P. (1977) Gene expression in a region of chromosome 17 during murine spermatogenesis. J. Immunogenet. 4, 353-362.

Erickson, R.P., Friend, D.S. and Tennenbaum, D. (1975a) The localization of lactate dehydrogenase-X on spermatozoa. Exp. Cell Res. 91, 1-5.

Erickson, R.P., Hoppe, P.C., Tennenbaum, D., Spielmann, H. and Epstein, C.J. (1975b) Lactate dehydrogenase $\mathrm{X}$ : effects of antibody on mouse gametes but not on early development. Science $188,261-263$.

Goldberg, E. and Lerum, J. (1972) Pregnancy suppression by an antiserum to the sperm specific lactate dehydrogenase. Science 176,686-687.

Nebel, B.R., Amarase, P.P. and Hackett, E.M. (1961) Calendar of gametogenic development of the prepuberal male mouse. Science 134, 832-833.

Nielsen, J.T. and Chapman, V.M. (1977) Electrophoretic variation for X-chromosome-linked phosphoglycerate kinase (PGK-1) in the mouse. Genetics 87, 319-325.

Pegaro, B., Ansari, A.A., Lee, C.-Y. and Erickson, R.P. (1978) Immunological relatedness of two isozymes of 3-phosphoglycerate kinase from the mouse. FEBS Letters 95, 371-374.

Pegaro, B. and Lee, C.-Y. (1978) Purification and partial characterization of two isozymes of 3-phosphoglycerate kinase from mouse. Biochim. Biophys. Acta 522, 423-433.

Pegaro, B. and Lee, C.-Y. (1979) Comparative biochemical studies of three variants of 3-phosphoglycerate kinase-2 in the mouse. Biochem. Genet. (in press).

Vandenberg, J.L., Cooper, D.W. and Close, P.J. (1973) Mammalian testis phosphoglycerate kinase. Nature New Biol. 243, 48-49.

Vandenberg, J.L., Cooper, D.W. and Close, P.J. (1976) Testis specific phosphoglycerate kinase B in mouse. J. Exp. Zool. 198, 231-240.

Yanagamachi, R., Yanagamachi, H. and Rogers, B.J. (1976) The use of zona-free animal ova as a test system for the assessment of the fertilizing capacity of human spermatozoa. Biol. Reprod. 15, $471-476$.

Yoyoda, Y. and Chang, M.C. (1974) Fertilization of rat eggs in vitro by epididymal spermatozoa and the development of the eggs following transfer. J. Reprod. Fert. 36, 9-22. 